La

Révolution

française

\section{La Révolution française}

Cahiers de l'Institut d'histoire de la Révolution française

$17 \mid 2020$

Gouverner par la loi

\title{
Les papiers de la Commission des Seize (frimaire- ventôse an III / décembre 1794-mars 1795)
}

\section{Céline Parcé}

\section{(2) OpenEdition}

\section{Journals}

Édition électronique

URL : http://journals.openedition.org/lrf/3502

DOI : $10.4000 /$ Irf.3502

ISSN : 2105-2557

Éditeur

IHMC - Institut d'histoire moderne et contemporaine (UMR 8066)

Référence électronique

Céline Parcé, « Les papiers de la Commission des Seize (frimaire-ventôse an III / décembre 1794-mars 1795) », La Révolution française [En ligne], 17 | 2020, mis en ligne le 24 février 2020, consulté le 20 avril 2020. URL : http://journals.openedition.org//rf/3502 ; DOI : https://doi.org/10.4000/Irf.3502

Ce document a été généré automatiquement le 20 avril 2020

(c) La Révolution française 


\title{
Les papiers de la Commission des Seize (frimaire-ventôse an III / décembre 1794-mars 1795)
}

\author{
Céline Parcé
}

1 Les archives produites par les assemblées depuis 1789 sont conservées dans la série $\mathrm{C}$ des Archives nationales, à l'exception de quelques ensembles de documents au nombre desquels figurent les papiers des comités de la période 1789-1795 ${ }^{1}$. Concernant ces comités, la série $\mathrm{C}$ comporte essentiellement les scrutins des élections de leurs membres sous la Constituante et la Législative, les états nominatifs et les bordereaux d'appointements de leurs employés et, de façon plus ponctuelle, les messages qu'ils ont adressés aux assemblées successives. Les registres d'enregistrement des lettres, adresses et pétitions reçues par les assemblées et transmises aux comités sont conservés dans la sous-série $\mathrm{C} / \mathrm{II}$ et les minutes de procès-verbaux des séances des assemblées dans la sous-série $\mathrm{C} / \mathrm{I}$. Pour retrouver les rapports imprimés présentés en séance par les comités et annexés à ces procès-verbaux, il faut se tourner vers la soussérie $\mathrm{AD} / \mathrm{XVIII}$.

2 L'intérêt principal de la série C pour l'étude des comités et de leurs rapports avec les commissions exécutives réside essentiellement dans un petit ensemble de documents distinct constitué des papiers de la Commission des Seize dont les travaux, encore peu étudiés, ont rarement été rapprochés de ceux aujourd'hui bien connus de la Commission des Onze qui lui a succédé. Ces archives, versées aux Archives nationales le 21 frimaire an IV (12 décembre 1795), comportent deux cartons ${ }^{2}$. On y trouve tout d'abord des documents relatifs à la composition de la Commission (arrêtés des comités désignant l'un des leurs pour les représenter en son sein) et les décrets de la Convention nationale la concernant (modalités de nomination de ses membres, renvois de propositions) $)^{3}$. Viennent ensuite plusieurs documents ou ensembles de documents rassemblés ou produits par la Commission dans le cadre de son activité. 


\section{La création de la Commission}

3 La Commission des Seize a été créée par la Convention nationale le 13 frimaire an III (3 décembre 1794) suite à une proposition du Comité des transports, postes et messageries visant à supprimer la commission exécutive du même nom et à la placer sous sa surveillance immédiate pour mettre fin à ses abus ${ }^{4}$. Dans son rapport présenté au nom du Comité, Garnier de Saintes justifie ce projet de décret en démontrant que les commissions «seraient plus sagement et plus économiquement remplacées par des agents responsables; qu'on ne peut surtout pas conserver ensemble des agences et des commissions qui, ayant un objet égal avec des pouvoirs inégaux, n'offrent qu'une rivalité de pouvoirs, une dépense énorme et des rouages inutiles au gouvernement ${ }^{5}$ ». Thibaudeau, s'il rejoint Garnier de Saintes sur la nécessité de mettre fin à ces dysfonctionnements et excès, rejette la proposition du Comité des transports, postes et messageries et demande le renvoi de la question aux comités :

Le projet de décret que l'on vous présente ne fait que changer le nom d'une administration sans en déraciner les abus. Il est des réflexions générales dont la Convention doit enfin s'occuper relativement aux commissions : je crois, moi, que le pouvoir est trop disséminé. Lorsque nous en avons décrété l'organisation, nous étions mus par une jalousie bien pardonnable sans doute envers la centralisation de ce pouvoir. La Convention doit méditer les moyens de resserrer le ressort qui doit faire agir le gouvernement. Il faut aussi porter un œil attentif sur l'organisation monarchique des bureaux des différentes commissions. Si l'on n'est pas revêtu d'un caractère public, on ne peut parvenir dans ces bureaux, ni finir ses affaires. L'organisation actuelle ne conviendrait qu'à un gouvernement qui ne voudrait point payer ses dettes. On consomme le temps et les deniers de la république en démolitions, en constructions, en distribution, en déménagements ; les employés se servent de ses meubles et de son linge. Une autre source des abus et des tiraillements du gouvernement se trouve dans la mauvaise administration de la commission de commerce et des approvisionnements. Eh! n'est-il pas ridicule de charger cinq individus d'approvisionner exclusivement vingt-cinq millions d'hommes! C'est le moyen d'avoir toujours une disette factice au milieu de l'abondance. Je conclus en demandant le renvoi du projet qu'on vient de proposer aux comités de gouvernement, afin qu'ils s'occupent des moyens de diminuer l'infâme bureaucratie qui nous dévore; d'empêcher les agents de la république d'étaler à ses dépens un faste scandaleux et de dilapider ses meubles, enfin pour qu'ils présentent une mesure plus utile et moins absurde que celle de la commission de commerce et des approvisionnements ${ }^{6}$.

4 Constituée de seize membres issus de chacun des comités de la Convention ${ }^{7}$, la Commission des Seize est ainsi créée afin d'étudier un nouveau mode d'organisation des commissions exécutives qui avaient remplacé les ministères à partir du 12 germinal an II ( $1^{\mathrm{er}}$ avril 1794). Elle se réunit entre le 21 frimaire (11 décembre 1794) et le 27 ventôse an III (17 mars 1795), au moment même où la Convention nationale débat des lois organiques visant à mettre en application la Constitution de 1793 et de la question, connexe, du maintien du gouvernement provisoire ${ }^{8}$.

\section{La documentation de travail de la Commission}

5 Alors que ses premiers travaux portent sur la question de savoir «s'il est plus avantageux de maintenir, de changer, ou de supprimer l'organisation actuelle des commissions exécutives et des agences ${ }^{9}$ » et sur « les mesures à prendre pour donner 
plus de ressort à l'action du gouvernement et en centraliser les mouvements ${ }^{10}$ ", la Commission se rend très vite compte qu'elle ne dispose pas des éléments suffisants pour y répondre et qu'elle doit tout d'abord acquérir une meilleure connaissance des attributions et $\mathrm{du}$ fonctionnement de chacune des commissions exécutives. Le 23 frimaire (13 décembre 1794), elle leur demande donc de lui adresser dans les trois jours un tableau détaillé de l'organisation de leurs bureaux et de leurs agences, par division, puis par subdivision. Elle-même s'organise en quatre bureaux pour étudier la documentation reçue ${ }^{11}$ et en rédiger des synthèses ${ }^{12}$. Volumineuse, celle-ci constitue la majeure partie des archives de la Commission des Seize. Elle est classée par bureau, puis par commission exécutive ou agence :

- Premier bureau :

- Commission de l'organisation et du mouvement des armées de terre

- Commission des armes et poudres

- Commission des travaux publics

- Commission des transports militaires, postes et messageries

- Deuxième bureau :

- Commission de la marine et des colonies

- Commission des relations extérieures

- Commission d'agriculture et des arts

- Troisième bureau :

- Commission du commerce et des approvisionnements

- Commission de l'instruction publique

- Commission des administrations civiles, police et tribunaux

- Agence de l'envoi des lois

- Quatrième bureau :

- Commission des revenus nationaux

- Trésorerie nationale

- Bureau de la comptabilité

- Liquidation générale

Le fonds ne comporte pas de dossier concernant la Commission des secours publics, qui était du ressort du deuxième bureau.

Qu'ils soient présentés sous la forme de mémoires rédigés ou de tableaux, les documents reçus sont extrêmement précis. Ils présentent en détail les attributions et le fonctionnement interne de chacune des commissions exécutives et des agences, mais aussi les relations qu'elles entretiennent les unes avec les autres, ainsi qu'avec les comités de la Convention. Ces tableaux sont accompagnés d'états nominatifs du personnel, les plus complets indiquant pour chacun des employés : son état civil, son adresse, ses emplois antérieurs avant et après la révolution, son traitement, ainsi que des observations sur sa moralité et sa façon de servir.

De tels documents avaient déjà été demandés aux commissions exécutives par la loi du 19 thermidor an II (6 août 1794), soit trois mois avant la création de la Commission des Seize. Regroupés au sein des papiers de la Commission, ils présentent l'avantage d'offrir un panorama des commissions exécutives et des agences dans leur ensemble (à l'exception de la Commission des secours publics) au tournant des mois de frimaire et de nivôse an III (novembre 1794-mars 1795).

En même temps qu'elle collecte cette documentation auprès des commissions exécutives, la Commission procède à l'audition d'anciens membres du Comité de salut 
public, les députés Bréard, Cochon, Lalloy, Robert-Lindet et Treilhard, qui sont invités, d'une part, à lui faire connaître les abus qu'ils ont pu constater dans l'organisation du gouvernement et des commissions exécutives et, d'autre part, à émettre des propositions pour la mise en place d'une nouvelle organisation du gouvernement capable d'assurer à la fois la direction des forces de la République à l'extérieur, le maintien de l'ordre à l'intérieur et l'exécution des lois. On en retrouve la trace dans le registre des délibérations qui fournit un résumé des débats, des points de vue et des décisions de la Commission, mais aussi de ces auditions ${ }^{13}$.

\section{Les projets de plans d'organisation du gouvernement pendant la Révolution}

Une fois ce travail de documentation préalable réalisé, la Commission des Seize engage à partir du 13 nivôse (2 janvier 1795) une discussion sur les réformes à faire dans le gouvernement ${ }^{14}$. Elle arrête à l'unanimité le 17 nivôse (6 janvier 1795) que le pouvoir exécutif doit être dans la représentation nationale :

La discussion est fixée sur la question de savoir si le Gouvernement sera placé dans la représentation nationale ou si on le placera hors de la Convention. Plusieurs membres obtiennent la parole successivement et discutent ces deux propositions. Ils font remarquer que dans l'état actuel des choses, et d'après l'imperfection où est encore la législation, il est impossible de former un conseil exécutif constitutionnel ou provisoire hors de la Convention qui réunisse assez de force et de moyens pour exercer les pouvoirs du Gouvernement et en diriger toutes les parties sans dangers. On établit au contraire par une multitude de motifs et de considérations la nécessité de concentrer dans la Convention les pouvoirs du Gouvernement qui seule peut les exercer avec avantage en les confiant à une portion de ses membres. On observe que par ces moyens, les forces et les relations de la République seront mieux ordonnées et dirigées au dehors, et l'ordre et la confiance maintenus dans l'intérieur, avec le concours des représentants dans les départements et aux armées $^{15}$.

11 La Commission se demande ensuite si le Gouvernement doit être concentré dans un seul comité ou s'il doit être réparti entre plusieurs ${ }^{16}$. Elle constitue un groupe de travail composé des députés Thibaudeau, Romme ${ }^{17}$ et Merlino chargés de réfléchir à cette question et de préparer un nouveau plan de gouvernement et d'organisation générale, tant des comités de la Convention nationale que des commissions exécutives, la Commission des Seize attribuant l'origine des dysfonctionnements du gouvernement à la dissémination des pouvoirs et à la mauvaise répartition des attributions entre les comités et les commissions exécutives ${ }^{18}$. On retrouve, au sein des archives de la Commission, les versions manuscrites successives du plan d'organisation du gouvernement pendant la Révolution proposées par ce groupe de travail ${ }^{19}$.

À partir du 16 ventôse (6 mars 1795), le registre des délibérations mentionne un second groupe de travail, constitué des députés Romme et Dumont (du Calvados), chargé d'examiner et de proposer ses vues sur les moyens qui pourront être employés pour préparer les lois organiques de la Constitution ${ }^{20}$. À l'issue de ses travaux, la Commission décide le 25 ventôse (15 mars 1795) de proposer à la Convention nationale la formation d'une commission particulière de neuf membres pour préparer et rédiger les lois organiques : ce sera la Commission des Onze. 
13 an III (19 mars 1795), lorsqu'elle est chargée par la Convention nationale de préparer, conjointement avec les Comités de salut public, de sûreté générale et de législation, « un projet de décret qui assure à jamais les droits des citoyens et la tranquillité de la République, conformément aux bases exprimées dans l'acte constitutionnel de mille sept cent quatre-vingt-treize ${ }^{21} »$.

Le 10 germinal an III (30 mars 1795), la Convention nationale crée la Commission des Sept qui doit proposer avant le $1^{\text {er }}$ floréal ( 20 avril) le mode à suivre pour préparer les lois organiques nécessaires à la mise en application de la Constitution de 1793. Suite à son rapport, la Commission des Onze est mise en place le 29 germinal an III (18 avril 1795). Cette dernière compte parmi ses membres un seul de ceux qui composaient la Commission des Seize : Thibaudeau. La Commission des Onze se charge de rédiger la nouvelle Constitution qui sera adoptée le 5 fructidor an III (22 août 1795).

\section{NOTES}

1. Une partie des archives du Comité des inspecteurs de la salle est toutefois classée dans la série C.

2. AN, C//355 et $\mathrm{C} / / 356$.

3. AN, C//355, dossiers 1870 et 1871 .

4. $\mathrm{AN}, \mathrm{C} / \mathrm{I} / 87$, Minute du procès-verbal de la séance de la Convention nationale du 13 frimaire an III (3 décembre 1794).

5. Gazette nationale ou Moniteur universel, $\mathrm{n}^{\circ}$ 75, 15 frimaire an III (5 décembre 1794).

6. Ibid.

7. Composition de la Commission des Seize au 14 frimaire an III (4 décembre 1794): Barras, Bidault, Boissier, Boucher-Sauveur, Cambacérès (remplacé par Richard), Danjou, Dubois, Dumont du Calvados, Fiquet (remplacé par Rouzet), Garnier de Saintes, Mailly, Merlino, Pérès, Réal, RouxFazillac (remplacé par Romme), Thibaudeau; après la recomposition du 28 pluviôse an III : Barras, Bion, Bodin, Boucher-Sauveur, Cambacérès, Danjou, Dumont du Calvados, Gouly, Laplaigne, Mailly, Merlino, Réal, Richard, Rivery, Romme, Thibaudeau.

8. Paolo COLOMBO, «LA QUESTION DU POUVOIR EXÉCUTIF DANS L'ÉVOLUTION INSTITUTIONNELLE ET LE DÉBAT POLITIQUE RÉVOLUTIONNAIRE », ANNALES HISTORIQUES DE LA RÉVOLUTION FRANÇAISE, № 319, 2000, P. 1-26.

9. AN, C//355 dossier 1872, Délibérations de la Commission des Seize du 23 frimaire an III (13 décembre 1794).

10. AN, C//355 dossier 1872, Délibérations de la Commission des Seize du 29 frimaire an III (19 décembre 1794).

11. Ibid.

12. AN, C//355, dossier 1889.

13. AN, C//355 dossier 1872, Délibérations de la Commission des Seize des 9 et 11 nivôse an III (29 et 31 décembre 1794).

14. AN, C//355 dossier 1872, Délibération de la Commission des Seize du 13 nivôse an III (2 janvier 1795).

La Révolution française, 17 | 2020 
15. AN, C//355 dossier 1872, Délibération de la Commission des Seize du 17 nivôse an III (6 janvier 1795).

16. Ibid.

17. Jean EHRARD, ALBERT SOBOUL (DIR.), «GILBERT ROMME ET SON TEMPS», ACTES DU COLLOQUE DE RIOM ET CLERMONT-FERRAND (10-11 JUIN 1965), PARIS, PRESSES UNIVERSITAIRES DE FRANCE, 1966; FRANÇOISE BRUNEL, « POURQUOI CES “SIX” PARMI LES “DERNIERS MONTAGNARDS” ? ", ANNALES HISTORIQUES DE LA RÉVOLUTION FRANÇAISE, No 304, AVRIL-JUIN 1996, P. 409.

18. AN, C//355 dossier 1872, Délibérations de la Commission des Seize du 13 nivôse au 28 pluviôse an III (2 janvier 1795-16 février 1795).

19. AN, C//356, dossier 1890. Michel TROPER, LA SÉPARATION DES POUVOIRS ET L'HISTOIRE CONSTITUTIONNELLE FRANÇAISE, PARIS, LIBRAIRIE GÉNÉRALE DE DROIT ET DE JURISPRUDENCE, 1973; MICHEL TROPER, TERMINER LA RÉVOLUTION. LA CONSTITUTION DE 1795, PARIS, FAYARD, 2006.

20. AN, C//355 dossier 1872, Délibérations de la Commission des Seize des 16 et 25 ventôse an III (6 et 15 mars 1795).

21. AN, C/I/90, Minute du procès-verbal de la séance de la Convention nationale du 29 ventôse an II (19 mars 1795).

\section{RÉSUMÉS}

La Commission des Seize est créée par la Convention nationale en frimaire an III (décembre 1794) pour réfléchir à un nouveau mode d'organisation des commissions exécutives, puis aux lois organiques nécessaires à la mise en application de la Constitution de 1793. Ses archives, conservées aux Archives nationales, constituent une source d'information extrêmement intéressante pour l'étude du fonctionnement interne des commissions exécutives et des relations qu'elles entretiennent les unes avec les autres ainsi qu'avec les comités de la Convention. Elles offrent également un aperçu des réflexions de la Commission sur l'organisation du gouvernement qui annoncent les travaux de la Commission des Sept, puis de la Commission des Onze, qui se verront confier ses attributions quelques mois plus tard.

The Commission of Sixteen was created by the National Convention in Frimaire Year III (December 1794) to develop a new mode of organisation of the executive commissions, and then define the organic laws necessary for the implementation of the Constitution of 1793. Its archives, held at the French National Archives, are an extremely interesting source of information for the study of the internal functioning of the executive commissions and their relations with each other and with the committees of the Convention. They also offer an insight into the reflections of the Commission on the organization of government on which will be based the work of the Committee of Seven, then the Committee of Eleven, which will be entrusted with its attributions a few months later. 
INDEX

Keywords : Commission of Sixteen, executive commissions, executive power, National Archives, French Revolution

Mots-clés : Commission des Seize, commissions exécutives, pouvoir exécutif, Archives nationales, Révolution française

\section{AUTEUR}

\section{CÉLINE PARCÉ}

Archives nationales 